\title{
High Selectivity Dual-Band Bandpass Filter with Tunable Lower Passband
}

\author{
Wei-Qiang Pan, Xiao-Lan Zhao, Yao Zhang, and Jin-Xu Xu \\ School of Electronic and Information Engineering, South China University of Technology, Guangzhou 510641, China
}

Correspondence should be addressed to Xiao-Lan Zhao; eexlzhao@126.com

Received 31 December 2014; Accepted 28 February 2015

Academic Editor: Dimitrios Sounas

Copyright (C) 2015 Wei-Qiang Pan et al. This is an open access article distributed under the Creative Commons Attribution License, which permits unrestricted use, distribution, and reproduction in any medium, provided the original work is properly cited.

This paper presents a novel method to design dual-band bandpass filters with tunable lower passband and fixed upper passband. It utilizes a trimode resonator with three controllable resonant modes. Discriminating coupling is used to suppress the unwanted mode to avoid the interference. Varactors are utilized to realize tunable responses. The bandwidth of the two bands can be controlled individually. Transmission zeros are generated near the passband edges, resulting in high selectivity. For demonstration, a tunable bandpass filter is implemented. Good agreement between the prediction and measurement validates the proposed method.

\section{Introduction}

The bandpass filters which are used to reject the unwanted signals have been widely investigated [1]. Meanwhile, to meet the requirement of the multiband operations, various methods have been proposed to design multiband bandpass filters [2-5]. Recently, electronically tunable bandpass filters have drawn much attention due to their capacity to deal with various operating frequencies in a system. Varactors are loaded at the ends of the resonators to adjust the operating frequencies [6]. Besides the frequency, the bandwidth of a tunable filter also should be taken into consideration [79]. In [7], suitable coupling region is selected to keep the bandwidth constant. Besides tunable single-band bandpass filters, tunable dual-band bandpass filters also have been widely researched [10-16]. In [11, 12], varactors are equipped at the center of stub-loaded open-ended resonator, resulting in a fixed lower passband and a tunable upper passband. To realize frequency tuning at both passbands, varactors are loaded not only at the center stub but also at the end of main transmission line [13]. For further convenient adjustment, two sets of resonators are employed [14-16]. By controlling different voltages, both of the two bands can be tuned.

However, most of the above references focus on designing dual-band filters with tunable upper passband, and few literatures address bandpass filters with a tunable lower passband and a fixed upper passband. The potential problem associated with this type of tunable filters is that the harmonic of the lower passband may overlap with the fixed upper passband.

To avoid this problem, we propose a novel method of using trimode resonators to design the tunable dual-band bandpass filter. The second mode (middle frequency) and third mode (highest frequency) of the trimode resonator are utilized to form the lower and upper passbands, respectively. The first mode (lowest frequency) is suppressed by discriminating coupling to eliminate the interference with the passband so that the selectivity can be enhanced. The second mode can be tuned by the varactors without affecting third mode, resulting in a tunable lower passband and fixed upper passband. Meanwhile, transmission zeros are synchronously changed with the passband, ensuring high skirt selectivity.

\section{Design Theory}

Figure 1(a) shows the proposed stub-loaded trimode resonator. It consists of a main transmission line and three open stubs. The characteristic admittance and the electronic length of each stub are shown. It is symmetric in structure; thus, even- and odd-mode method can be used to characterize it and its odd- and even-mode equivalent circuits are shown as in Figures 1(b) and 1(c). Since Figures 1(b) and 1(c) are still 


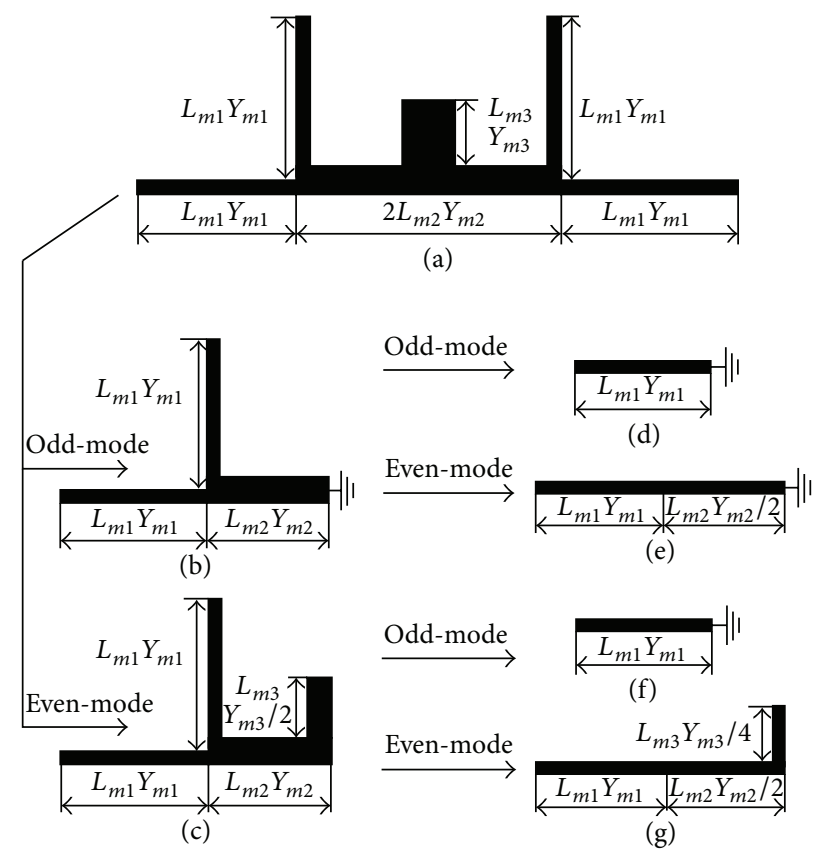

FIGURE 1: (a) The proposed stub-loaded trimode resonator; (b) odd-mode circuit of (a); (c) even-mode circuit of (a); (d) odd-mode circuit of (b); (e) even-mode circuit of (b); (f) odd-mode circuit of (c); (g) even-mode circuit of (c).

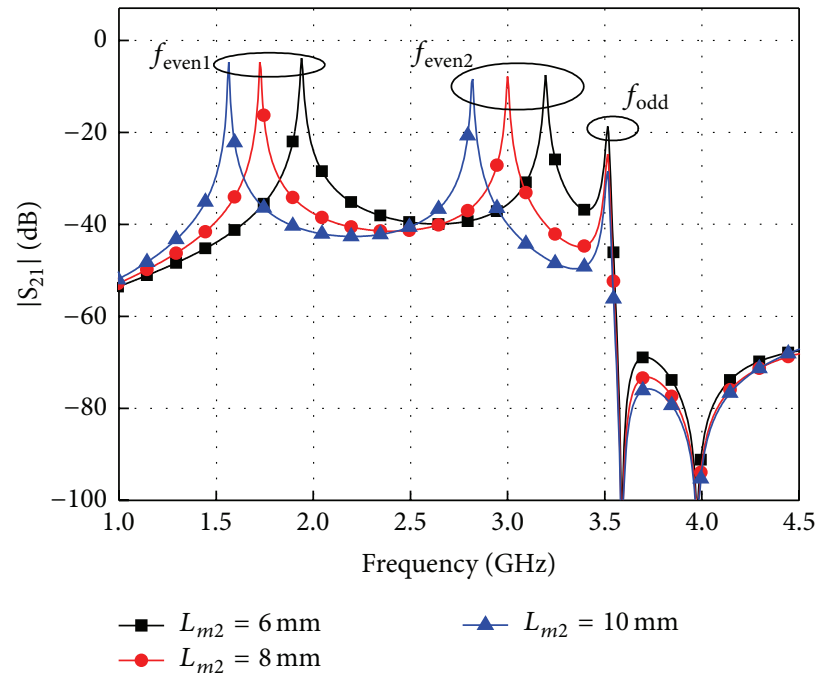

(a)

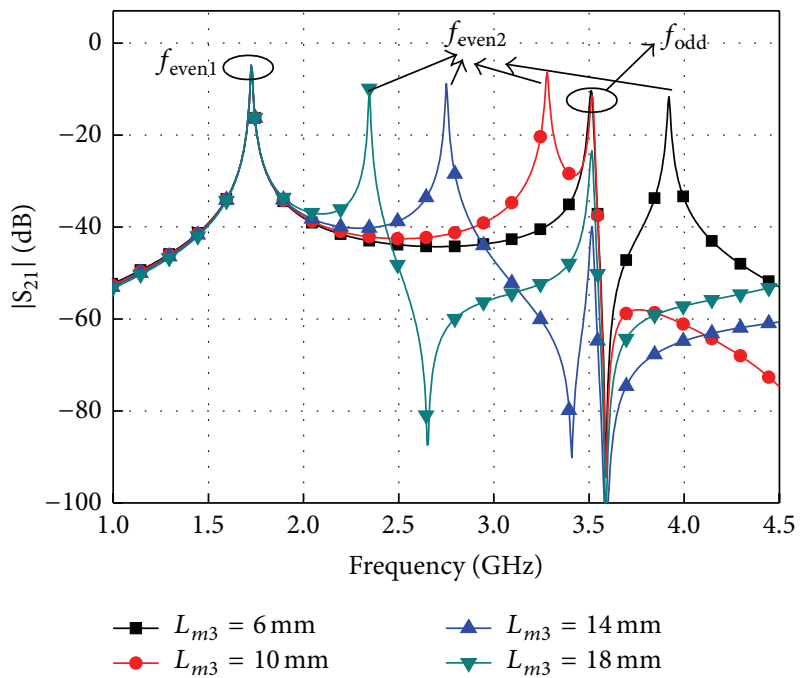

(b)

FIgURE 2: Simulated $\left|\mathrm{S}_{21}\right|$ against (a) $L_{m 2}$; (b) $L_{m 3}$.

symmetric, even- and odd-mode method can be further used to analyze them. The corresponding equivalent circuits are shown as Figures $1(\mathrm{~d})-1(\mathrm{~g})$. Under the special condition of $Y_{m 3}=2 Y_{m 2}=4 Y_{m 1}$, the resonant frequencies of Figures 1(d), $1(\mathrm{e}), 1(\mathrm{f})$, and $1(\mathrm{~g})$ can be deduced as follows [2]:

$$
\begin{gathered}
f_{\text {odd } 1}=\frac{c}{4 L_{m 1} \sqrt{\varepsilon_{\text {eff }}}}, \\
f_{\text {even1 }}=\frac{c}{4\left(L_{m 1}+L_{m 2}\right) \sqrt{\varepsilon_{\text {eff }}}},
\end{gathered}
$$

$$
\begin{gathered}
f_{\text {odd } 2}=\frac{c}{4 L_{m 1} \sqrt{\varepsilon_{\text {eff }}}}, \\
f_{\text {even2 }}=\frac{c}{2\left(L_{m 1}+L_{m 2}+L_{m 3}\right) \sqrt{\varepsilon_{\text {eff }}}},
\end{gathered}
$$

where $c$ is the light speed in free space and $\varepsilon_{\text {eff }}$ denotes the effective dielectric constant of the substrate. Comparing the four frequencies, $f_{\text {odd } 1}$ and $f_{\text {odd2 } 2}$ are the same, and we 


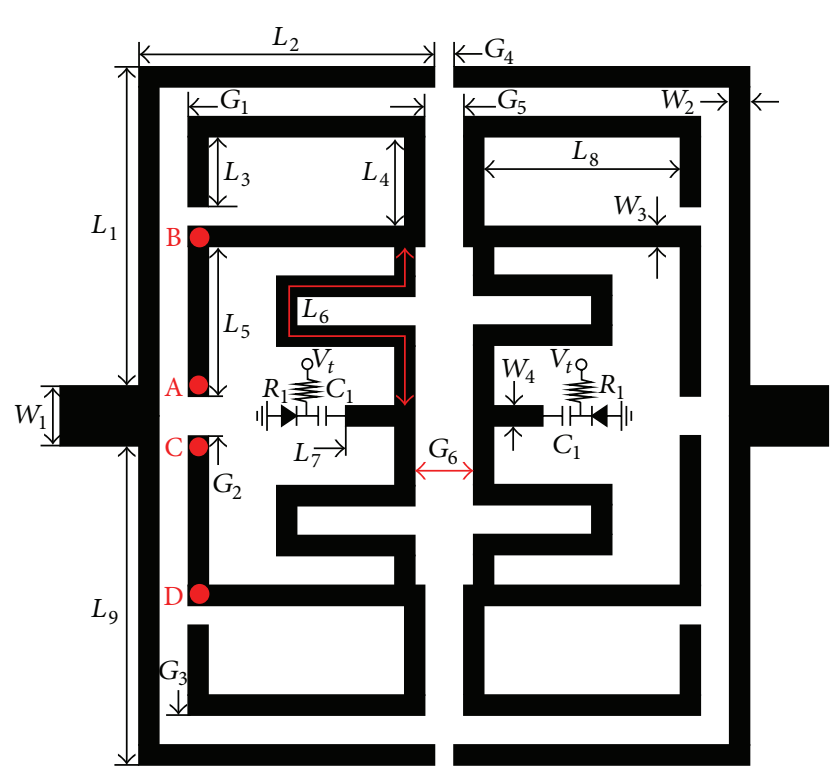

FIGURE 3: Structure of the proposed tunable dual-band filter.

use $f_{\text {odd }}$ to denote them. Meanwhile, we can obtain $f_{\text {odd }}>$ $f_{\text {even } 1}$, while the relationship of $f_{\text {even2 }}$ and $f_{\text {odd }}$ could not be determined since it is related to $L_{m 3}$. Small $L_{m 3}$ results in $f_{\text {even2 }}>f_{\text {odd }}$ while large $L_{m 3}$ results in $f_{\text {even2 }}<$ $f_{\text {odd }}$. Moreover, it can be observed that $L_{m 3}$ only affects $f_{\text {even2 }}$ without affecting $f_{\text {even1 }}$ and $f_{\text {odd }} . L_{m 2}$ affects both $f_{\text {even2 }}$ and $f_{\text {even1 }}$ without affecting $f_{\text {odd }}$. Thus, the trimode can be controlled individually. To demonstrate this, some simulations are carried out by using ADS. In the simulations, the parameters are chosen as follows: $L_{m 1}=13.3 \mathrm{~mm}, L_{m 2}=$ $8 \mathrm{~mm}, L_{m 3}=6 \mathrm{~mm}, Y_{m 1}=Y_{m 2}=Y_{m 3}=0.01 \mathrm{~S}$, and $\varepsilon_{r}=$ 3.38. When one parameter is swept, the other parameters are fixed. The simulated results are shown in Figure 2. It can be observed that when $L_{m 2}$ is changed, $f_{\text {even1 }}$ and $f_{\text {even2 }}$ are also changed while $f_{\text {odd }}$ is fixed. When $L_{m 3}$ is changed, only $f_{\text {even } 2}$ is changed and the other two modes are fixed. Thus, we can design a dual-band filter with tunable lower passband which is formed by $f_{\text {even2 }}$ (the second mode) and fixed upper passband formed by $f_{\text {odd }}$ (the highest mode) and suppressed $f_{\text {even1 }}$ (the lowest mode).

Figure 3 shows the structure of the proposed dual-band filter with tunable lower passband. It utilizes a second order stub-loaded trimode resonator and the stubs are folded to reduce the size. The varactors are loaded at the center stubs. The capacitor $C_{1}(2 \mathrm{pF})$ is used to block the DC signals. The $R_{1}$ with the high resistance of $47 \mathrm{k} \Omega$ can prevent the RF signals and is used as DC feed. The feed lines are coupled with the resonators. There is an important issue that should be noticed: the feed line should be mirror symmetrical with respect to the port and the center of the resonator. With such a structure, the mode of $f_{\text {even } 1}$ can be suppressed and the tunable lower passband and fixed upper passband can be realized.

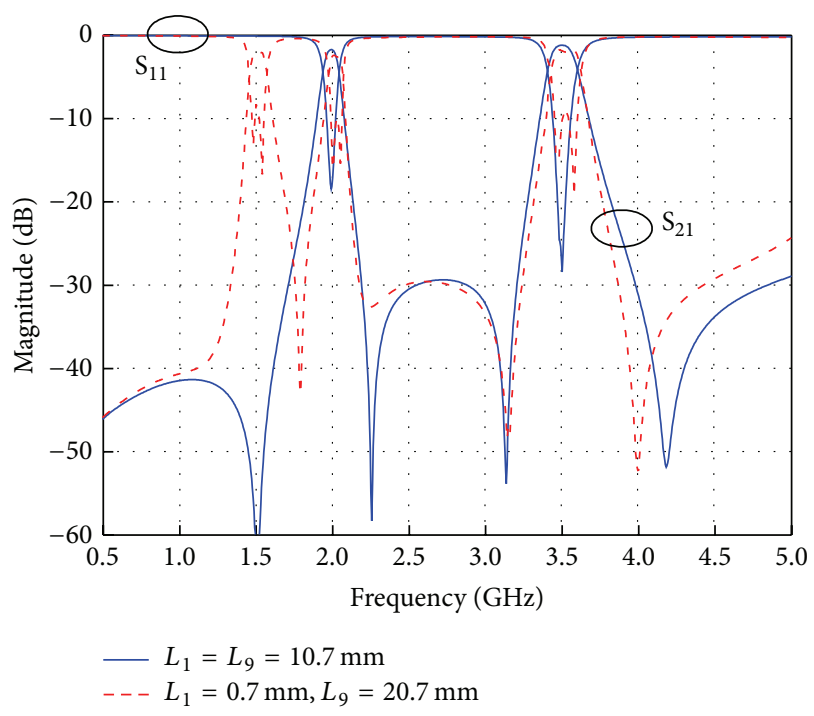

FIGURE 4: Simulated results under the condition of symmetric and asymmetric port position.

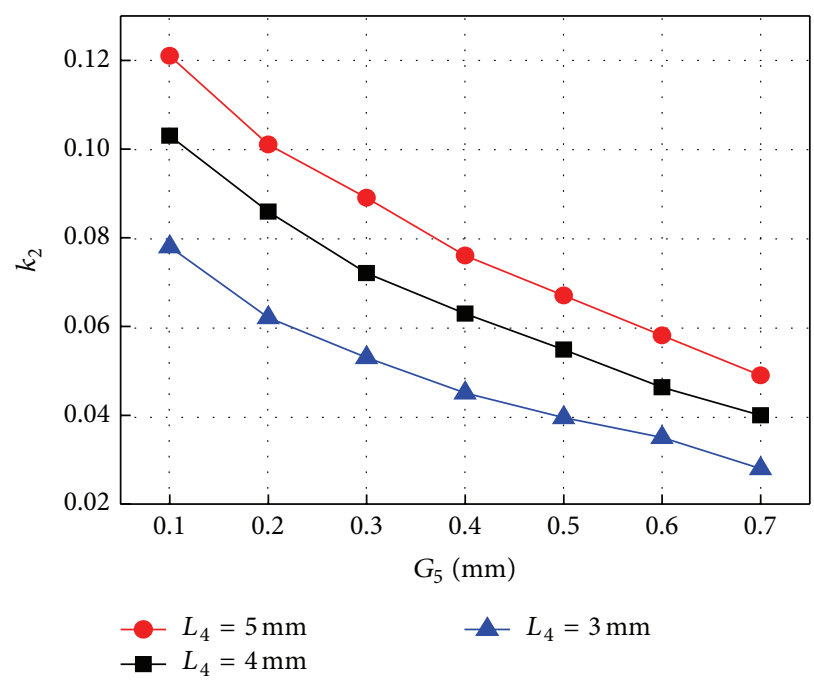

Figure 5: $k_{2}$ against $G_{5}$ and $L_{4}$.

The mechanism of $f_{\text {even1 }}$ suppression is addressed as follows. For a parallel coupled microstrip line with a length of $d$, the electric coupling can be approximated as [17]

$$
k_{e}=p \times \frac{\int_{-d / 2}^{d / 2} V_{1}(x) V_{2}(x) d x}{\sqrt{\int_{-d / 2}^{d / 2}\left|V_{1}(x)\right|^{2} d x \times \int_{-d / 2}^{d / 2}\left|V_{2}(x)\right|^{2} d x}},
$$

where $V_{1}(x)$ and $V_{2}(x)$ are voltage-wave functions on the two lines and $p$ represents a constant. When the voltagewave function is odd on one line and even on the other, the integrand in the numerator is an odd function and it yields a zero value upon integration, which implies that the electric coupling coefficient is zero. Similarly, the magnetic coupling coefficient is also zero. Thus, no energy can pass at this frequency, resulting in mode suppression. 


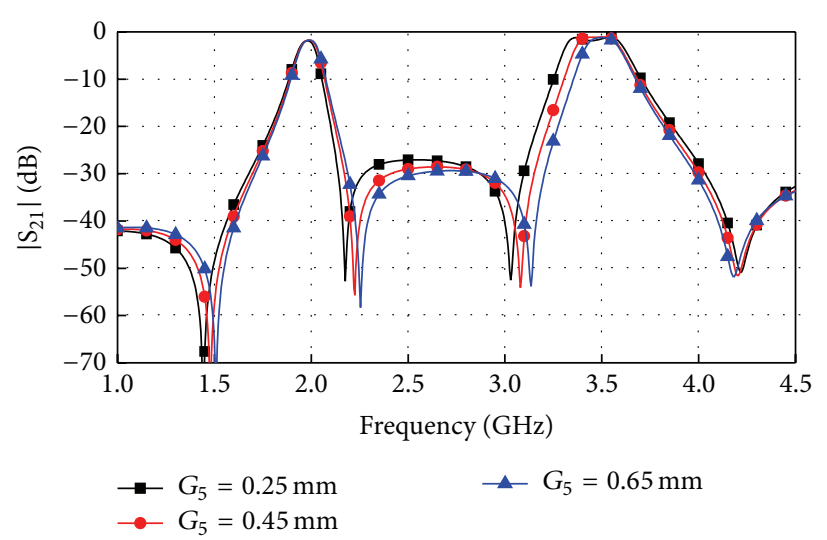

(a)

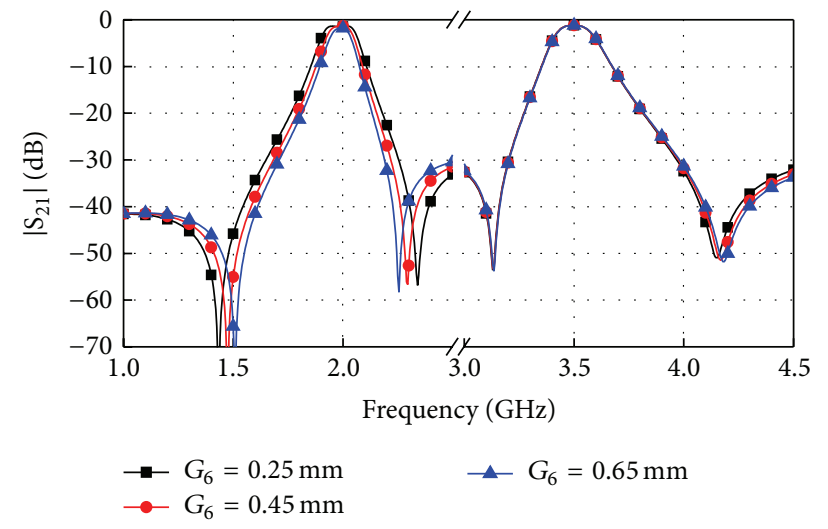

(b)

FIGURE 6: Simulated $\left|S_{21}\right|$ against (a) $G_{5}$; (b) $G_{6}$ (the varactors are deleted and replaced by capacitors with value of $3 \mathrm{pF}$ ).

According to the analysis, $f_{\text {even } 1}$ is determined by $L_{m 1}$ and $L_{m 2}$. In Figure 3, if the mode is not suppressed, it corresponds to a passband which is affected by $2\left(L_{5}+L_{6}+L_{8}\right)+W_{4}$. It is a half-wavelength resonator and the voltage distribution at the two open ends (AB and $\mathrm{CD}$ ) with regard to the feed point is odd symmetrical. However, the voltage distribution at the two sides of feed line with regard to the feed point is even symmetrical. Thus, according to (2), the mode of $f_{\text {even } 1}$ is suppressed without affecting $f_{\text {even2 }}$ and $f_{\text {odd }}$. In the suppression mechanism, the key point is the mirror symmetry of the resonator and the feed line with regard to the feed point. To demonstrate this, simulations are carried out under the condition of the port at and not at the center of the feed line. To observe the modes, the varactors are replaced by capacitors. Figure 4 shows the simulated results. It can be observed that when the port is not located at the center of the feed line there will be three passbands, corresponding to the mode: $f_{\text {even } 1}, f_{\text {even } 2}$ and $f_{\text {odd }}$. If the port is located at the center of the feed line, the first passband will be suppressed and the other two bands are kept unchanged. Moreover, the selectivity of the lower passband is improved. Using such a feed method, the $f_{\text {even } 1}$ can be suppressed without any extra circuit.

To design a tunable dual-band bandpass filter, we can first design a dual-band filter with fixed centre frequencies of $f_{1}$ and $f_{2}$. The frequencies and bandwidths can be individually controlled as follows. For the operating frequencies, it can be controlled individually. We can first obtain $f_{2}$ by tuning the length of $L_{5}+L_{8}$, which is nearly quarter-wavelength at $f_{2}$. Then, $L_{6}$ and $L_{7}$ are used to control $f_{1}$ without affecting $f_{2}$. For the bandwidth, it is determined by the external quality factor $\left(Q_{e}\right)$ and coupling coefficient $(k)$. For the $Q_{e}$, it is determined by the coupling lengths and gaps, for example, $L_{1}, L_{2}, G_{1}$, and $G_{2}$. Small gaps $\left(G_{1}\right.$ and $\left.G_{2}\right)$ and large lengths $\left(L_{1}\right.$ and $\left.L_{2}\right)$ result in small $Q_{e}$ and large bandwidths at both passbands. For $k$, it is determined by the coupling strength between the two resonators, for example, $G_{5}$ and $G_{6}$. Since $L_{6}$ has little effect on $f_{2}, G_{6}$ can be used to control $k$ at $f_{1}$ with little effect on $f_{2}$. To determine the initial value of these parameters, we can use simulation software to extract $k_{2}$.

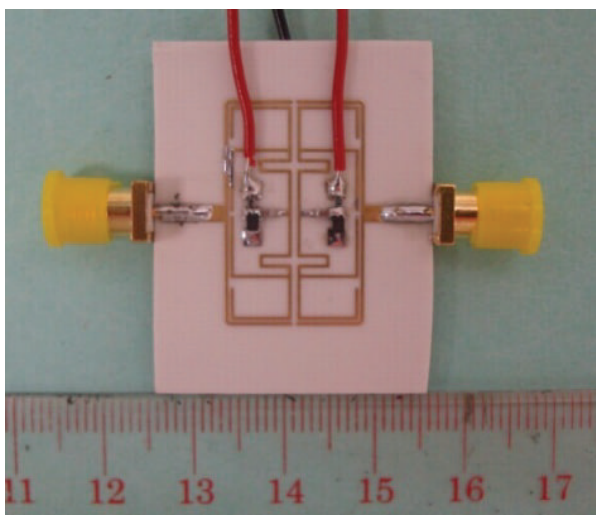

FIGURE 7: Photograph of the fabricated circuit.

The second passband is fixed with $3 \mathrm{~dB}$ bandwidth of $6 \%$ and $0.5 \mathrm{~dB}$ ripple. Thus, the required $k_{2}$ is 0.06 . Figure 5 shows the extracted $k_{2}$ against $G_{5}$ and $L_{4}$. Using this figure, the $G_{5}$ and $L_{4}$ can be determined as 0.2 and $3 \mathrm{~mm}$. Similarly, the initial value of $G_{6}$ can be determined as $0.3 \mathrm{~mm}$. Figure 6 shows the simulated results against $G_{5}$ and $G_{6}$. It can be observed that when $G_{5}$ increases, the bandwidth of upper passband decreases and the bandwidth of the lower passband decreases. When $G_{6}$ is changed, only the bandwidth of lower passband changes and the upper passband is fixed. These results indicate that the bandwidth of the two bands can be controlled individually. After the nontunable filter is welldesigned, varactors are loaded at the end of $L_{7}$ to realize tunable responses. Then, fine tuning is used to obtain the optimal performance.

\section{Experiment}

To demonstrate the proposed idea, a circuit is implemented on the substrate with a relative dielectric constant of 3.38, loss tangent of 0.0027 , and thickness of $0.81 \mathrm{~mm}$. The varactors employed in this design are 1sv277 from Toshiba. The capacitors are from Murata and we use the Murata model 


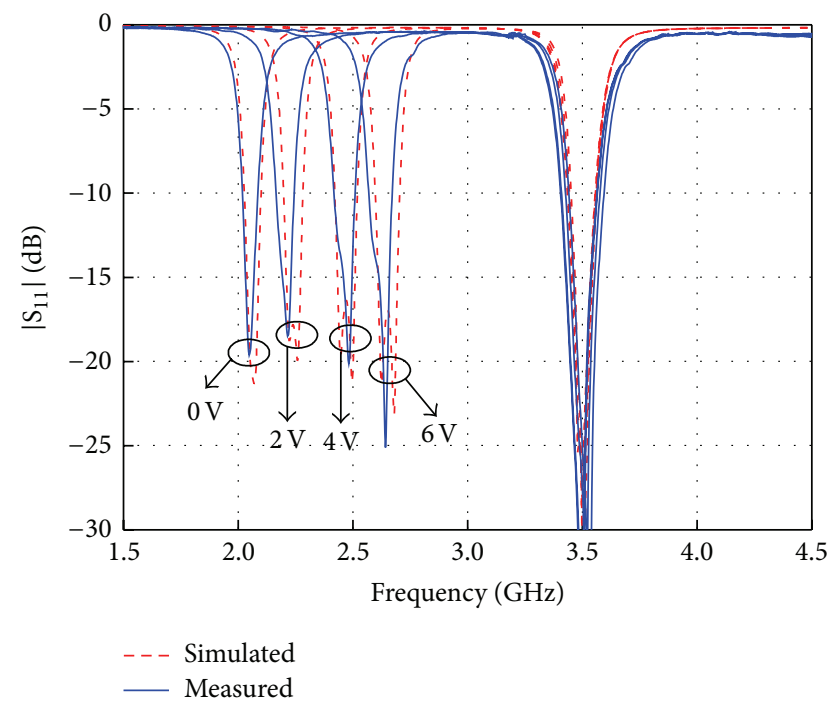

(a)

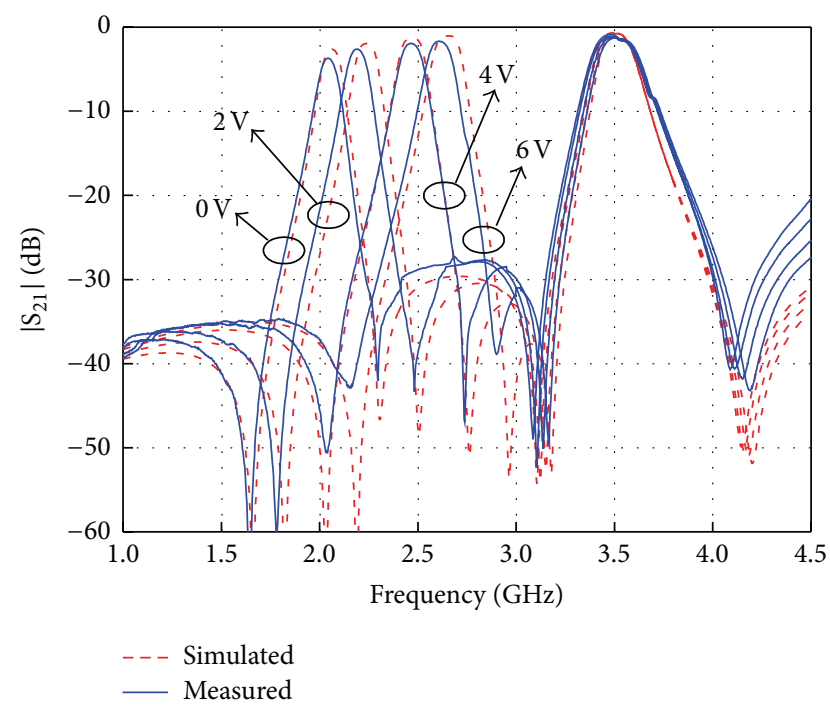

(b)

FIGURE 8: Simulated and measured results (a) $S_{11}$ and (b) $S_{21}$.

in simulation. The final values are selected as follows (all in $\mathrm{mm}): L_{1}=10.7, L_{2}=6.9, L_{3}=2.5, L_{4}=4, L_{5}=6.5$, $L_{6}=11.4, L_{7}=1.9, L_{8}=5.7, L_{9}=10.7, W_{1}=1.86$, $W_{2}=W_{3}=W_{4}=0.4, G_{1}=0.2, G_{2}=1, G_{3}=0.2$, $G_{4}=0.3, G_{5}=0.65$, and $G_{6}=0.65$. And $R_{1}=47 \mathrm{k} \Omega$; $C_{1}=2 \mathrm{pF}$. The overall size of the filter is $14.9 \mathrm{~mm} \times 24 \mathrm{~mm}$ or $0.16 \lambda_{g} \times 0.27 \lambda_{g}$, where $\lambda_{g}$ represents the guided wavelength at $2.04 \mathrm{GHz}$, which is the lowest frequency of the tunable passband. The photograph of the fabricated filter is shown in Figure 7.

The simulation and measurement are accomplished by IE3D, ADS, and 8753ES network analyzer, respectively. Figure 8 shows the simulated and measured results. The upper passband is fixed at $3.5 \mathrm{GHz}$ with $3 \mathrm{~dB}$ bandwidth of $200 \mathrm{MHz}$ or $5.7 \%$, which covers the WiMAX system. The measured minimum insertion loss is $1.5 \mathrm{~dB}$ and the return loss is better than $30 \mathrm{~dB}$. Two transmission zeros with the frequencies of 3.15 and $4.25 \mathrm{GHz}$ are generated at both sides of the passband. For the lower passband, with the voltage increasing from 0 to $6 \mathrm{~V}$, the operating frequency changes from 2.04 to $2.62 \mathrm{GHz}$ with $3 \mathrm{~dB}$ bandwidth changing from 90 to $135 \mathrm{MHz}$. The return losses are better than $15 \mathrm{~dB}$ in each state. The insertion losses change from $3.7 \mathrm{~dB}$ to $1.7 \mathrm{~dB}$, which is a little higher than the nontunable bandpass filter. It is mainly due to the loss of the varactors. Meanwhile, the two transmission zeros located at the two sides of the lower passbands synchronously change with the passband, ensuring high selectivity. Among the four transmission zeros, the first one is generated by the source-load coupling; the second and last ones are generated by the stub with length of $L_{m 3}$ and $L_{m 1}$. The third one is introduced by the feed line and $L_{1}+L_{2}$ is quarter-wavelength at the frequency of the third transmission zero.

\section{Conclusion}

This paper has presented a novel method for designing dual-band bandpass filters with a tunable lower passband and fixed upper passband. Both theory and experiments have been provided, showing that the filter exhibits good performance. Transmission zeros are generated near the passbands, resulting in high selectivity.

\section{Conflict of Interests}

The authors declare that there is no conflict of interests regarding the publication of this paper.

\section{Acknowledgments}

This work is supported by the NSFC under Grant 61271209 and the Fundamental Research Funds for the Central Universities under Grant 2013ZM0106.

\section{References}

[1] Y.-L. Lu, S. Wang, T. Gu, P. Cao, and K. Li, "A miniaturize bandpass filter with harmonic suppression using meandered quarterwavelength resonators," International Journal of Antennas and Propagation, vol. 2014, Article ID 916927, 6 pages, 2014.

[2] L. Gao and X. Y. Zhang, "High-selectivity dual-band bandpass filter using a quad-mode resonator with source-load coupling," IEEE Microwave and Wireless Components Letters, vol. 23, no. 9, pp. 474-476, 2013.

[3] F.-C. Chen, Q.-X. Chu, and Z.-H. Tu, "Tri-band bandpass filter using stub-loaded resonators," Electronics Letters, vol. 44, no. 12, pp. 747-749, 2008. 
[4] Y. Liu, W. B. Dou, and Y. J. Zhao, "A tri-band bandpass filter realized using tri-mode T-shape branches," Progress in Electromagnetics Research, vol. 105, pp. 425-444, 2010.

[5] H. Chen, "High isolation tri-band BPF using tri-mode resonator," Microwave and Optical Technology Letters, vol. 55, no. 6, pp. 1367-1370, 2013.

[6] C. Ge and X.-W. Zhu, "High-selective tunable bandpass filter with two-path mixed coupling," IEEE Microwave and Wireless Components Letters, vol. 24, no. 7, pp. 451-453, 2014.

[7] X.-G. Huang, Q.-Y. Feng, Q.-Y. Xiang, and D.-H. Jia, “Constant absolute bandwidth tunable filter using varactor-loaded openloop resonators," Microwave and Optical Technology Letters, vol. 56, no. 5, pp. 1178-1181, 2014.

[8] Y.-Y. Wang, F. Wei, B. Liu, H. Xu, and X.-W. Shi, "A tunable bandpass filter with constant absolute bandwidth based on one ring resonator," Journal of Electromagnetic Waves and Applications, vol. 26, no. 11-12, pp. 1587-1593, 2012.

[9] B. Liu, F. Wei, H. Zhang, X. Shi, and H. Lin, "A tunable bandpass filter with switchable bandwidth," Journal of Electromagnetic Waves and Applications, vol. 25, no. 2-3, pp. 223-232, 2011.

[10] L. Gao, X. Y. Zhang, B.-J. Hu, and Q. Xue, "Novel multi-stub loaded resonators and their applications to various bandpass filters," IEEE Transactions on Microwave Theory and Techniques, vol. 62, no. 5, pp. 1162-1172, 2014.

[11] X. Y. Zhang and Q. Xue, "Novel centrally loaded resonators and their applications to bandpass filters," IEEE Transactions on Microwave Theory and Techniques, vol. 56, no. 4, pp. 913-921, 2008.

[12] L. Zhang, X.-H. Wang, Z.-D. Wang, Y.-F. Bai, and X.-W. Shi, "Compact electronically tunable microstrip dual-band filter using stub-loaded SIRs," Journal of Electromagnetic Waves and Applications, vol. 28, no. 1, pp. 39-48, 2014.

[13] X. Huang, L. Zhu, Q. Feng, Q. Xiang, and D. Jia, "Tunable bandpass filter with independently controllable dual passbands," IEEE Transactions on Microwave Theory and Techniques, vol. 61, no. 9, pp. 3200-3208, 2013.

[14] G. Chaudhary, Y. Jeong, and J. Lim, "Dual-band bandpass filter with independently tunable center frequencies and bandwidths," IEEE Transactions on Microwave Theory and Techniques, vol. 61, no. 1, pp. 107-116, 2013.

[15] X. Y. Zhang, L. Gao, Y. Cao, X.-L. Zhao, and Y. Ding, "Independently-tuned dual-band filter using varactor-loaded resonators," Progress In Electromagnetics Research C, vol. 42, pp. 55-66, 2013.

[16] B. You, L. Chen, Y. Liang, and X. Wen, "A high-selectivity tunable dual-band bandpass filter using stub-loaded steppedimpedance resonators," IEEE Microwave and Wireless Components Letters, vol. 24, no. 11, pp. 736-738, 2014.

[17] X. Y. Zhang and Q. Xue, "Harmonic-suppressed bandpass filter based on discriminating coupling," IEEE Microwave and Wireless Components Letters, vol. 19, no. 11, pp. 695-697, 2009. 

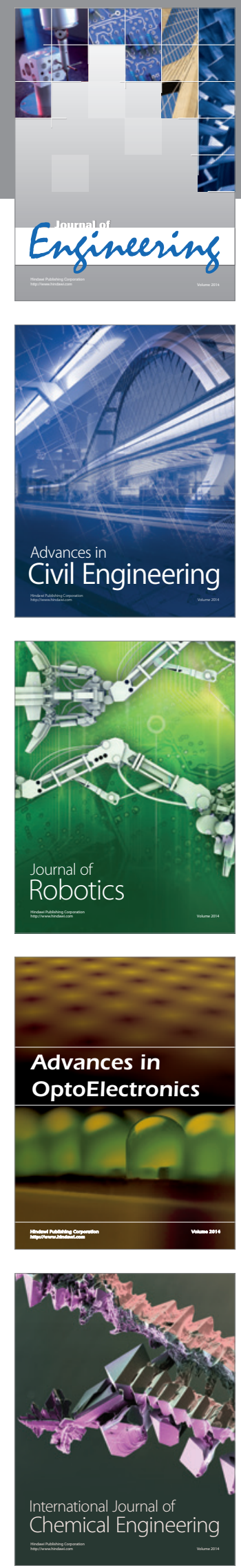

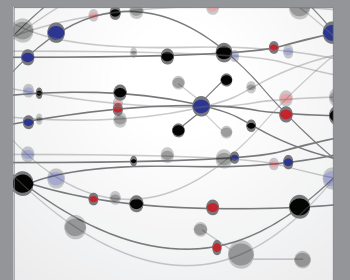

The Scientific World Journal
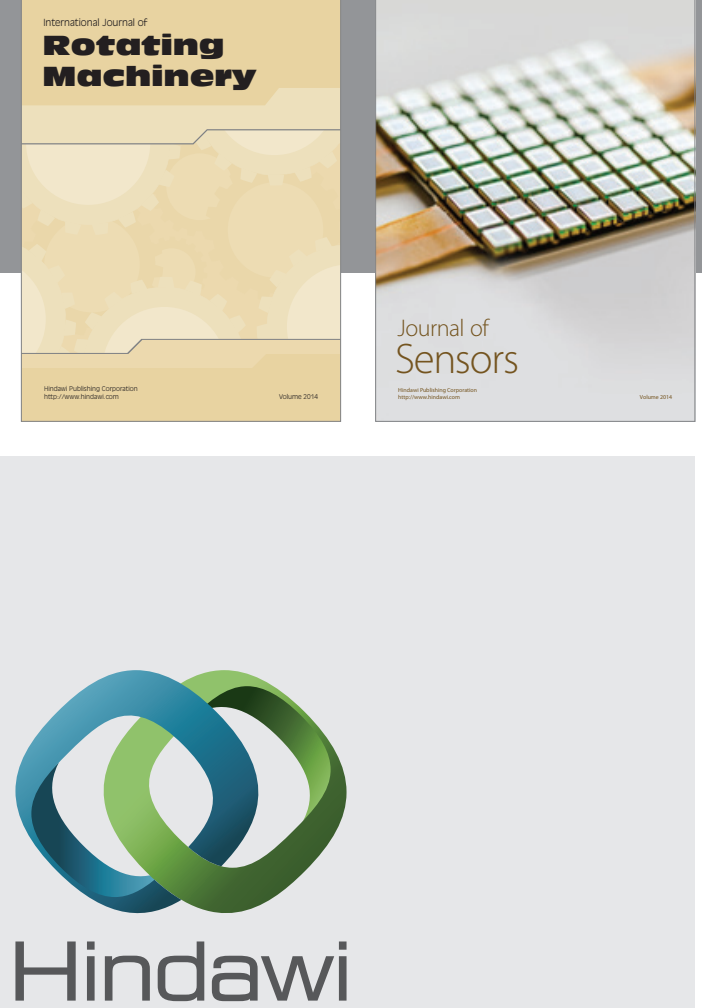

Submit your manuscripts at http://www.hindawi.com
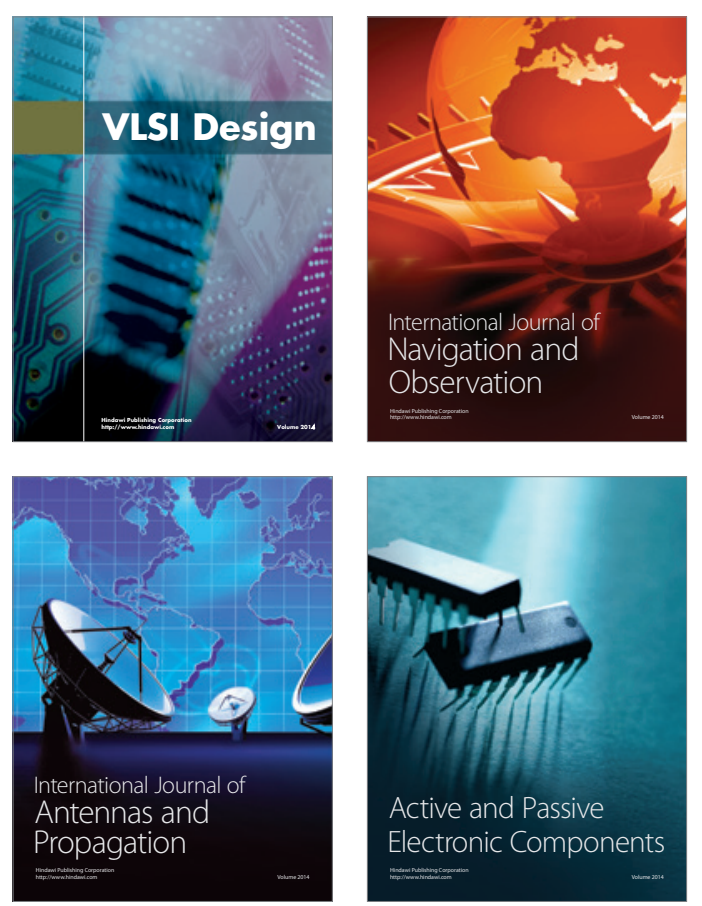
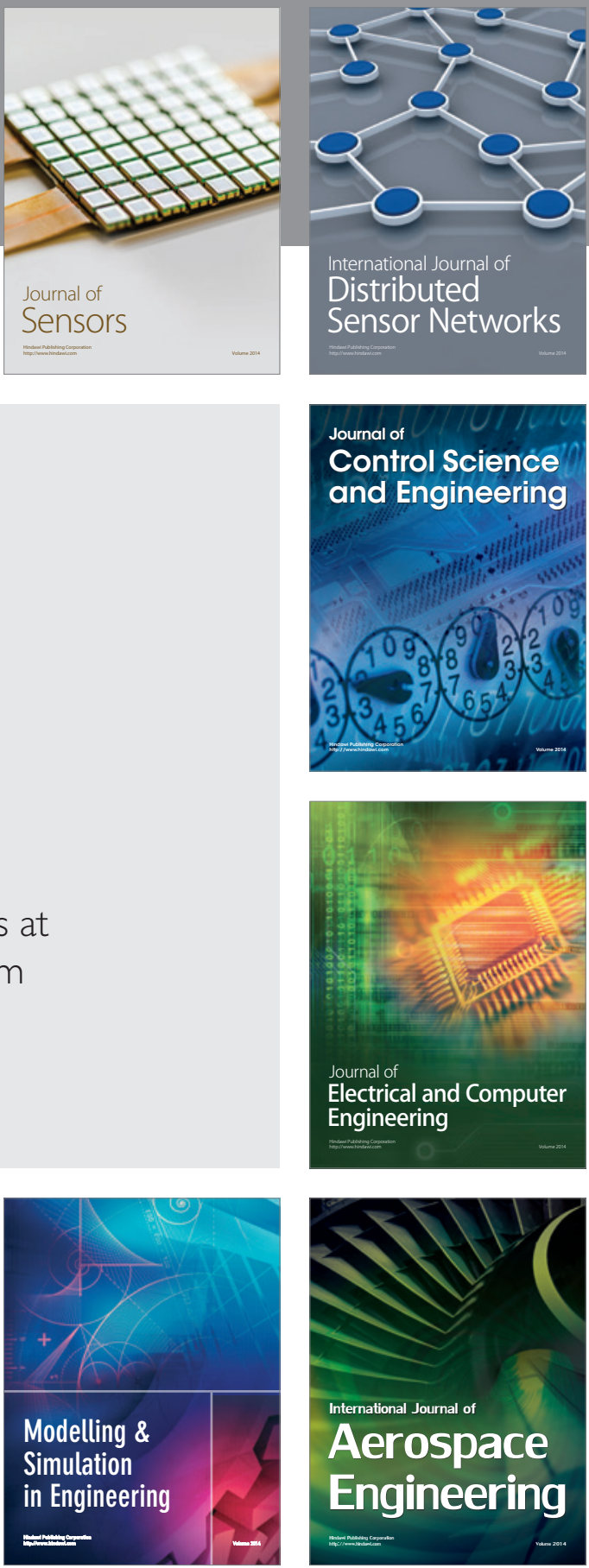

Journal of

Control Science

and Engineering
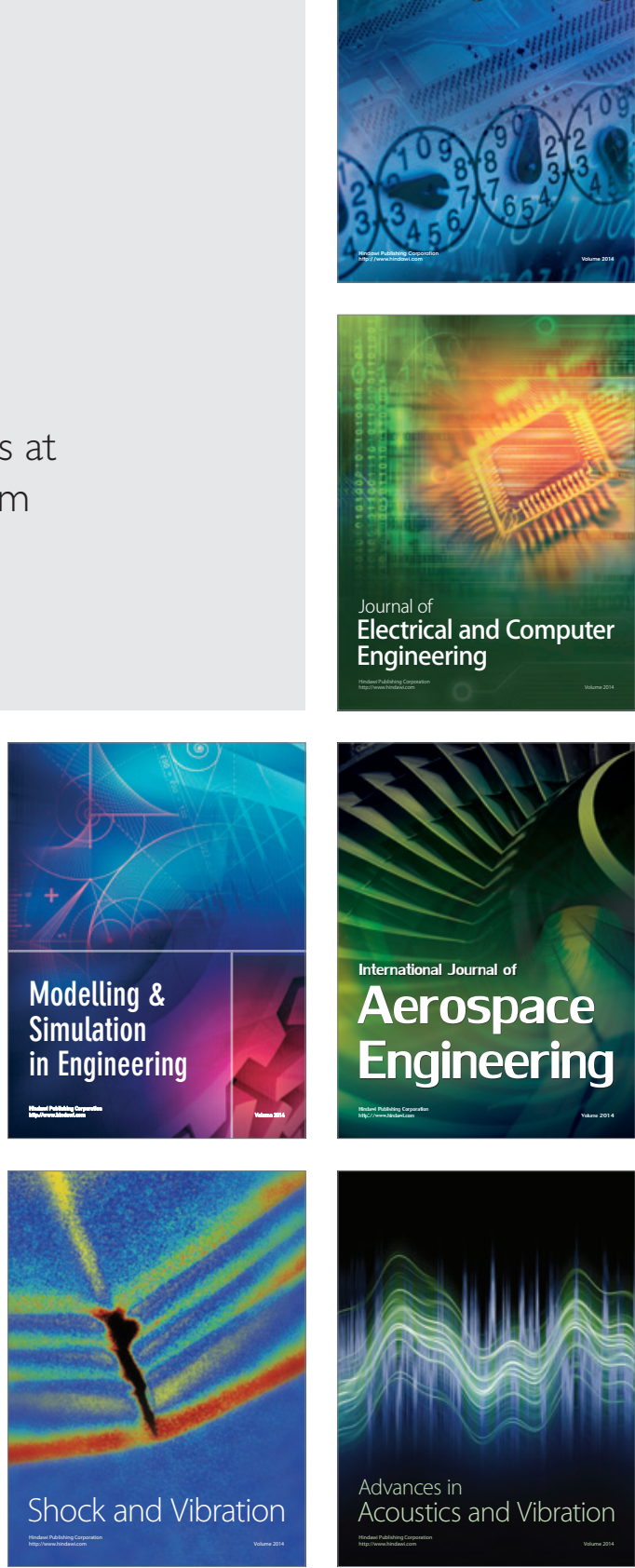